\title{
Drug treatment of toxoplasmic encephalitis in acquired immunodeficiency syndrome
}

Peter Winstanley

\section{Summary}

Toxoplasma gondii is a ubiquitous parasite, which causes several serious diseases. Until the AIDS pandemic, toxoplasmic encephalitis was largely confined to the pharmacologically immunesuppressed and to cases of congenital transmission. $T$ gondii is now one of the more commonly encountered opportunistic pathogens of advanced AIDS.

Keywords: HIV/AIDS, cerebral toxoplasmosis, chemotherapy

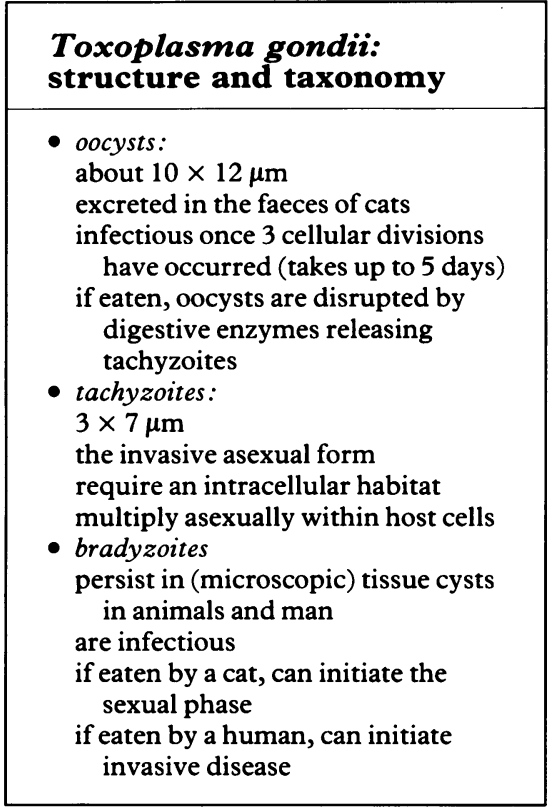

Box 1

\section{The organism}

Toxoplasma gondii, an obligate intracellular parasite, belongs to the Class Sporozoa and the Order Coccidia. There is more than one strain of the organism, and strains vary in virulence, surface antigens, isozymes and drug-sensitivity. Patients with toxoplasmosis are not infectious, and need not be isolated.

\section{Epidemiology}

The prevalence rate of seropositivity for $T$ gondii increases with age, and varies geographically: up to $90 \%$ of adults are seropositive in France and El Salvador, compared with about $10-40 \%$ in the UK and the US. It has been suggested that this variation may result from dietary habits.

\section{Pathogenesis}

$T$ gondii spreads by invading and disrupting contiguous cells, and by dissemination in nucleated blood cells. Tachyzoites proliferate, causing local necrosis and stimulating a mononuclear cell reaction. Even so, in immunocompetent people, acute toxoplasmosis may go unnoticed - it is symptomatic in only $20 \%$ of cases.

Eventually, such acute infection becomes controlled by host defences including non-specific cellular mechanisms (including macrophages and natural killer cells), specific cellular immunity (cytokines, including $\gamma$-interferon, seem to have a major role) and humoral immunity (IgG, IgM, IgA and IgE).

Tachyzoites have the ability to 'transform' into bradyzoites, which are contained within tissue cysts. This parasite stage is able to elude host defences, and may remain viable for many years as an asymptomatic latent infection. Cysts can be found in any tissue, but are common in brain, striated muscle and the heart. From time to time, bradyzoites may 'escape' from cysts, without disruption of the cyst wall. In the immune-competent host this 'leakage' may give rise to 'daughter cysts'. In immuno-suppressed patients, however, such escape may give rise to re-activation of the latent infection.

The commonest site for re-activation is the central nervous system (CNS), although disease may occur elsewhere, such as the lungs. It is unclear whether CNS disease results from disruption of tissue cysts locally, or whether there is haematogenous spread of tachyzoites from other sites. The CNS lesions comprise patchy meningoencephalitis and necrosis (often due to vascular involvement), and these result in the clinical syndrome known as toxoplasmic encephalitis (figure 1).

\section{Clinical features of toxoplasmic encephalitis}

\section{SYMPTOMS AND SIGNS}

Toxoplasmic encephalitis may complicate immunosuppression of any cause, but is nowadays most commonly seen in advanced acquired immunodeficiency syndrome (AIDS), most patients having low CD4 counts (below $200 \times 10^{6} / 1$ in $90 \%$ of cases). Estimates of the proportion of AIDS sufferers who develop toxoplasmic encephalitis varies, but is probably between $5 \%$ and $30 \%$. Most patients have IgG antibodies to $T$ gondii, suggesting re-activation of latent disease; new infection (suggested by an IgM response) is much less common.

The speed of onset varies from insidious to acute. Common presenting symptoms and signs are listed in box 3. 


\begin{tabular}{|l|}
\hline Routes of infection \\
\hline - oral: by eating undercooked meat \\
(mainly lamb or pork; tissue cysts \\
are rarely found in cattle); infection \\
is common, and undetectable by \\
naked-eye inspection \\
- faecal-oral spread from cats: oocysts \\
may be transferred to the mouth \\
after handling cats \\
transplacental: infection of a \\
non-immune mother carries the \\
risk of transplacental spread and \\
foetal damage \\
- miscellaneous: blood transfusion, \\
organ donation (particularly heart), \\
or laboratory accidents \\
\hline
\end{tabular}

Box 2

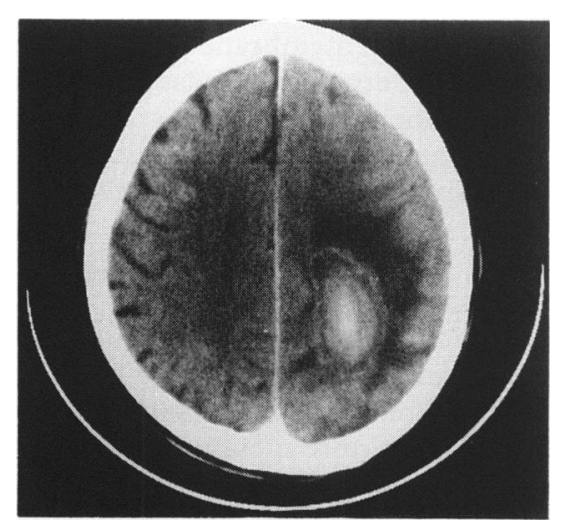

Figure 1 Enhanced brain CT scan showing a ring-enhancing lesion in an AIDS patient with toxoplasmosis

\begin{tabular}{|l|}
\hline \multicolumn{2}{|l|}{ Common symptoms and } \\
signs
\end{tabular}

Box 3

\section{INVESTIGATION}

Clinical suspicion is best investigated by computed tomography (CT) or magnetic resonance imaging (MRI) - the latter giving superior definition of small lesions. CNS lesions are multiple in $73 \%$ of cases, and most frequent in the frontal cortex, basal ganglia and parietal cortex. Sometimes a solitary lesion may be seen on CT (figure 1); MRI is recommended in such patients to search for previously overlooked small lesions, and thereby help distinguish toxoplasmic encephalitis from other intracranial pathology, notably lymphoma. ${ }^{2}$

Other investigations are less helpful. The presence of specific IgG antibodies demonstrates previous infection with $T$ gondii; however IgG antibodies do not prove re-activation of the disease, and their absence does not exclude it. Demonstration of an IgM response suggests recent infection, but this is unusual. Lumbar puncture may be hazardous in the face of mass lesions, and the cerebrospinal fluid is often normal (though there may be mild elevations in pressure, protein concentration and cell count). T gondii can be cultured from the blood in about $10 \%$ of cases, but this figure is too low to be of practical value. The role of sensitive techniques for detection of circulating parasite DNA, by polymerase chain reaction, is still being assessed. Brain biopsy, although often diagnostic, is complicated by false negative results and can cause morbidity. It is therefore reserved for diagnostic problems: Luft and Remington ${ }^{2}$ recommend biopsy for patients with a solitary lesion on MRI (not CT), and those who show no evidence of improvement after 14 days antiprotozoan therapy.

\section{DIFFERENTIAL DIAGNOSIS}

Differential diagnoses include other infectious causes of meningoencephalitis (tuberculosis, fungi and viruses, especially Herpes simplex), brain abscess, stroke, progressive multifocal leucoencephalopathy and tumours (especially lymphoma). ${ }^{3}$

\section{PROGNOSIS}

In AIDS patients, mean survival after diagnosis is about one year. ${ }^{1}$ Deep coma at presentation, previous Kaposi sarcoma, previous Pneumocystis carinii pneumonia, high fever and a lymphocyte count lower than $24 \%$ of the differential are poor prognostic factors.

\section{Prevention}

Between $25 \%$ and $50 \%$ of AIDS patients seropositive for $T$ gondii, will develop toxoplasmic encephalitis - usually when their CD4 count has fallen below $200 / \mathrm{mm}^{3}$. Effective primary prophylaxis for such patients would be of benefit. Use of cotrimoxazole, as prophylaxis against $P$ carinii pneumonia, may confer benefit as may the combination of pyrimethamine with dapsone. However, the use of prophylactic pyrimethamine in addition to cotrimoxazole may increase death rate $-a$ reminder that these drugs are far from innocuous.

\section{Drugs used for treatment and maintenance}

\section{PYRIMETHAMINE}

Mode of action (figure 2)

The conversion of 2 'deoxyribosyluracil monophosphate to 2 'deoxyribosylthymine monophosphate is essential for normal DNA synthesis in mammals and protozoa; this is accomplished by donation of a methyl group by methylenetetrahydrofolate and the process is catalysed by thymidylate synthetase. The oxidised product, dihydrofolate, must be reduced back to replenish the 'pool' of methylene tetrahydrofolate: the rate-limiting step of this process is catalysed by dihydrofolate reductase, and pyrimethamine competes with dihydrofolate for this enzyme. Pyrimethamine is active against tachyzoites, but ineffective against tissue cysts; it is unknown whether this is due to failure of pyrimethamine to cross the cyst wall, or because of differences between bradyzoites and tachyzoites.

Pharmacokinetics (box 4)

Pyrimethamine ${ }^{4}$ is available only as tablets, although Fansidar (pyrimethamine plus sulfadoxine; Roche) does have an intramuscular formulation. Pyrimethamine is rapidly and extensively absorbed when given orally to patients with malaria, but absorption from intramuscular Fansidar is slower. ${ }^{5}$ The extent and rate of absorption of oral pyrimethamine by AIDS sufferers with toxoplasmic encephalitis seems to be highly variable. ${ }^{6}$ Pyrimethamine is widely distributed to the tissues, and concentrates in brain tissue. The drug is eliminated 


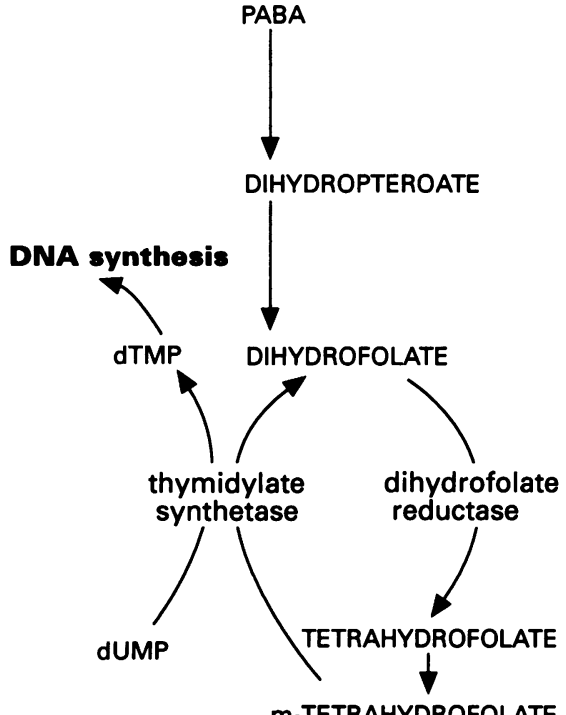

m-TETRAHYDROFOLATE

Figure 2 The folate pathway in protozoa, dTMP $=2$ 'deoxyribosylthymine monophosphate; dUMP $=2$ 'deoxyribosyluracil monophosphate; PABA = para-aminobenzoic acid

\begin{tabular}{|l|}
\hline $\begin{array}{l}\text { Pharmacokinetics of } \\
\text { pyrimethamine }\end{array}$ \\
\hline - well absorbed (from the gut) by \\
healthy subjects \\
oral absorption by AIDS patients \\
may be variable \\
- widely distributed, and \\
concentrates in brain \\
- half-life (in healthy adults) between \\
46 and $150 \mathrm{~h}$ \\
\hline
\end{tabular}

Box 4

\begin{tabular}{|l|}
\hline $\begin{array}{l}\text { Adverse effects of } \\
\text { pyrimethamine }\end{array}$ \\
\hline - bone marrow suppression \\
- photosensitive rash \\
- acute gout \\
\hline
\end{tabular}

Box 5

\begin{tabular}{|l|}
\hline $\begin{array}{l}\text { Pharmacokinetics of } \\
\text { sulfadiazine }\end{array}$ \\
\hline - well absorbed (from the gut) by \\
healthy subjects \\
oral absorption by AIDS patients \\
not fully studied \\
- widely distributed, crosses the \\
blood:brain barrier \\
- half-life $6-12 \mathrm{~h}$ \\
Box 6
\end{tabular}

Box 6 (very slowly) by hepatic biotransformation to inactive metabolites. Because the elimination half-time is about $100 \mathrm{~h}$, pyrimethamine accumulates when given daily.

\section{Clinical use}

Pyrimethamine is usually given in synergistic combination with sulfadiazine, clindamycin, ${ }^{4}$ or dapsone. The standard dose regimen is $200 \mathrm{mg}$ followed by $50-100 \mathrm{mg}$ daily until four weeks after a clinical response is seen. Clinical response to pyrimethamine combinations can take up to 15 days, about $10 \%$ of patients fail to respond, $10-20 \%$ relapse during maintenance therapy and up to $60 \%$ develop adverse drug reactions. Maintenance therapy (25-50 mg daily) is mandatory, since tissue cysts are not eradicated and recurrence is almost certain if maintenance is omitted. During treatment of active cerebral toxoplasmosis, pyrimethamine levels should probably exceed $750 \mathrm{ng} / \mathrm{ml}$ (when in combination with either sulfadiazine or clindamycin). ${ }^{6}$

\section{Adverse effects (box 5)}

Patients given daily pyrimethamine accumulate the drug, eventually achieving high plasma concentrations which may affect the bone marrow, skin and mucous membranes. Marrow toxicity may manifest as megaloblastic anaemia, neutropaenia or thrombocytopaenia. Skin and mucous membrane effects may manifest as glossitis, stomatitis, and exfoliative dermatitis. Folate-deficient patients are at particular risk of concentration-dependent toxicity, and folinic acid [leucovorin] may be helpful in treatment and prevention. Idiosyncratic reactions are rare, although HIV-positive patients may sometimes develop a rash.

\section{OTHER DIHYDROFOLATE REDUCTASE INHIBITORS}

Other dihydrofolate reductase inhibitors include biguanides and methotrexate analogues. The biguanides, such as proguanil and chlorproguanil are used for malaria prevention and treatment, and have a reputation for safety. Both proguanil and chlorproguanil are pro-drugs which require metabolism to their pharmacologically active triazine metabolites, cycloguanil and chlorcycloguanil, for therapeutic effects to be seen; neither is routinely used for toxoplasmic encephalitis. However, an extremely potent analogue, WR250417, is under development, which may have utility. WR250417 (also known as PS-15) is converted to the triazine WR99210 - one of the most potent antimalarial drugs known. Combination of WR250417 with either a sulphonamide or a sulphone seem to be efficacious against both $T$ gondii and $P$ carinii.

The methotrexate analogues, trimetrexate and piritrexim, are effective against $T$ gondii infections, and have been used clinically. However, both are limited by haematological toxicity.

\section{SULFADIAZINE AND OTHER SULPHONAMIDES}

\section{Mode of action (figure 2)}

Sulphonamides inhibit the incorporation of para-aminobenzoic acid into dihydropteroate, a precursor of dihydrofolate, by competitively inhibiting the enzyme dihydropteroate synthetase which is an enzyme of many micro-organisms (including bacteria and Plasmodia) but is not possessed by mammals. Against $T$ gondii sulfadiazine is synergistic with pyrimethamine at lower concentration than other sulphonamides, and is therefore preferred. Other sulphonamides with reasonable activity against $T$ gondii include sulphamethoxazole and sulphadimidine; in contrast, sulfadoxine has very low potency.

\section{Pharmacokinetics (box 6)}

The disposition of sulfadiazine in patients with advanced HIV-disease has not been thoroughly studied. In healthy subjects sulfadiazine seems to be rapidly absorbed from the gut, mainly from the small intestine: peak plasma levels are achieved within four hours of oral dosing. An intravenous formulation is available for use in patients with impaired consciousness. Sulfadiazine is widely distributed throughout the body: concentrations in brain tissue have not been measured, but those in the cerebrospinal fluid (CSF) are about $70 \%$ those in the plasma. Sulfadiazine is eliminated mainly by acetylation; the rate of this process is genetically determined, individuals being classed as rapid or slow acetylators. Both sulfadiazine and (inactive) metabolite are excreted in the urine, but much sulfadiazine is reabsorbed by the tubules; sulfadiazine clearance is increased in the presence of alkaline urine, and decreased in renal failure. The elimination half-time of sulfadiazine is $6-12 \mathrm{~h}$ in those with normal renal and hepatic function. 


\begin{tabular}{|l|}
\hline $\begin{array}{l}\text { Adverse effects of } \\
\text { sulfadiazine }\end{array}$ \\
\hline - allergic reactions ranging from mild \\
to life-threatening \\
- crystalluria \\
- haemolysis in glucose-6-phosphate- \\
dehydrogenase deficiency
\end{tabular}

Box 7
Clinical use

Sulfadiazine is given in synergistic combination with pyrimethamine; $75 \mathrm{mg} \mathrm{kg}^{-1}$ (up to $4 \mathrm{~g}$ ) is usually given as a loading dose, followed by $100 \mathrm{mg} \mathrm{kg}^{-1}$ daily (up to $8 \mathrm{~g}$ daily in 2-4 doses); this is continued for six weeks, and is followed by a maintenance dose of $2 \mathrm{~g}$ daily in divided doses. Sulfadiazine may be given orally or intravenously.

Adverse effects (box 7)

Crystalluria can complicate the use of sulfadiazine, especially in acid urine, but the most common severe adverse effects are idiosyncratic. Serum sickness, interstitial nephritis, rashes (including erythema multiforme), hepatitis and blood dyscrasias are not uncommon in HIV-positive patients, and necessitate withdrawal of sulfadiazine in a high proportion of patients. Why allergic reactions to sulphonamides are so common in AIDS patients remains unknown, although deficiency in glutathione and acquired impairment of drug acetylation in this group have been suggested as contributory factors. In patients with glucose-6-phosphate-dehydrogenase deficiency sulphonamides may precipitate severe haemolysis, causing haemoglobinuria and acute renal failure.

\section{CLINDAMYCIN AND SIMILAR ANTIBIOTICS}

Mode of action

Clindamycin, azithromycin, spiramycin, roxithramycin, and clarithromycin, which are thought to work by interference with ribosomal protein synthesis, all have activity against tachyzoites of $T$ gondii. The greatest experience has been gained with clindamycin, an antibiotic which may be given by oral, intramuscular or intravenous routes.

\section{Pharmacokinetics}

Clindamycin is rapidly absorbed when given orally or intramuscularly and reaches maximum concentration within two hours. The drug's bioavailability appears to be higher in patients with advanced HIV-disease than in normal subjects, possibly because of impaired first-pass metabolism. Clindamycin is widely distributed, but its penetration into the CSF is not extensive. The drug is eliminated mainly by hepatic metabolism (some of the metabolites retain pharmacological activity) and is mainly excreted in the bile. The elimination half-time in healthy subjects is about three hours, this can be modestly prolonged in the presence of severe liver disease.

\section{Clinical use}

Clindamycin is usually combined with pyrimethamine, being the usual recourse in patients with toxoplasmosis who are allergic to sulphonamides. Pyrimethamine/clindamycin seems to be about as effective as pyrimethamine/ sulfadiazine, ${ }^{4}$ though data are incomplete.

\section{Adverse effects}

In the dosages used $(1.2 \mathrm{~g}$ six hourly intravenously for up to three weeks, followed by $300 \mathrm{mg}$ six hourly by mouth thereafter) toxicity from clindamycin is common (box 8); quinidine-like cardiotoxicity is a special problem if the drug is given rapidly intravenously.

\section{Other macrolides and lincosamines}

Of the newer macrolides, azithromycin is probably the most potent, and also seems to have activity against bradyzoites in tissue cysts: its role is still being studied.

- quinidine-like cardiotoxicity causing ventricular fibrillation

- deranged liver function, and overt hepatitis

- rashes

\section{ATOVAQUONE}

Because of the poor risk:benefit ratios of existing drugs, and the increasing frequency of toxoplasmic encephalitis in late AIDS, new chemotherapeutic agents are under investigation. Perhaps the most promising is atovaquone, an hydroxynaphthoquinone inhibitor of mitochondrial ubiquinones. Atovaquone was developed as an antimalarial drug, but also has potent antitoxoplasma activity against both tachyzoites and bradyzoites. Atovaquone synergises with sulphonamides and pyrimethamine. Although atovaquone is not yet generally available, the manufacturer will often provide it on a 'named-patient basis'. 
1 Porter S, Sande M. Toxoplasmosis of the central nervous system in the acquired immunodeficiency syndrome. $N$ Engl $\mathscr{f}$ Med 1992; 327 1643-8

2 Luft BJ, Remington JS. Toxoplasmic encephalitis in AIDS. Clin Infect Dis 1992; 15: 211-22.

3 McCabe RE, Remington JS. Toxoplasma gondii. In: Mandell GL, Douglas RG, Bennett JE, eds. Principles and practice of infectious diseases. 3rd edn. New York: Churchill Livingstone, 1990.
4 Dannemann B and the California Collaborative Treatment Group. Treatment of toxoplasmic encephalitis in patients with AIDS: a randomised trial comparing pyrimethamine plus clindamycin to pyrimethamine plus sulfadiazine. Ann Intern Med 1992; 116: 33-43.

5 Winstanley PA, Watkins WM, Newton CRJC et al. The disposition of oral and intramuscular pyrimethamine/sulfadoxine in Kenyan children with high parasitaemia but clinically non-severe falciparum malaria. Br $f$ Clin Pharmacol 1992. 33: $143-8$.
6 Weiss LM, Harris C, Berger M, Tanowitz HB, Wittner M. Pyrimethamine concentrations in serum and cerebrospinal fluid during treatmen of acute toxoplasma encephalitis in patients with AIDS. $\mathcal{f}$ Infect Dis 1988; 157: 580-3.

\section{Medical Anniversary \\ JONATHAN HUTCHINSON, 23 JULY 1828}

Jonathan Hutchinson (1828-1913) was born at Selby, Yorkshire, UK, into a Quaker family. After early education at Selby and the York School of Medicine, he studied at St Bartholomew's Hospital where he came under the influence of Sir James Paget.

Hutchinson earned the title of the most famous general practitioner in the world because of his wide range of interests and appointments. He was dermatologist at the Blackfriars Hospital for Diseases of the Skin; Ophthalmologist to the Royal London Ophthalmic Hospital; Venereologist to the Lock Hospital; Physician to the City of London Chest Hospital; and General Surgeon to the London and Metropolitan Hospitals. In addition to his Presidency of the Hunterian Society (1869) he was founder-secretary of the New Sydenham Society (1859-1907); President of the Royal College of Surgeons (1889); President of the Pathological Society of London (1879); President of the Ophthalmological Society of the United Kingdom (1883); President of the Neurological Society (1887); President of the Medical Society of London (1892); President of the Royal Medical and Chirurgical Society (1894-6); and President of the 1896 International Dermatology Congress. 